\title{
Estresse no Trabalho e Associação ao Consumo de Ultraprocessados por Servidores Universitários
}

\author{
Bianca de Oliveira Farias ${ }^{1}$, Ana Carolina Montenegro Cavalcante ${ }^{2}$, Rafaella Maria Monteiro Sampaio ${ }^{3}$, \\ Maria Yasmin Paz Teixeira ${ }^{4}$, Helena Alves de Carvalho Sampaio ${ }^{5}$, Soraia Pinheiro Machado ${ }^{6}$
}

\footnotetext{
${ }^{1}$ http://orcid.org/0000-0001-6678-3763 / Universidade Estatual do Ceará (UECE), Brasil

2 http:/ / orcid.org/0000-0002-1086-0587 / Universidade de Fortaleza (UNIFOR), Brasil

${ }^{3}$ http://orcid.org/0000-0001-9994-1916/ Universidade de Fortaleza (UNIFOR), Brasil

${ }^{4}$ http://orcid.org/0000-0002-2054-6284 / Universidade Estatual do Ceará (UECE), Brasil

${ }^{5}$ http://orcid.org/0000-0001-5353-8259 / Universidade Estatual do Ceará (UECE), Brasil

${ }^{6}$ http://orcid.org/0000-0002-3918-4738 / Universidade Estatual do Ceará (UECE), Brasil
}

Resumo

O estresse no trabalho afeta cada vez mais a saúde do trabalhador, sobretudo seus hábitos alimentares. Ainda, há crescente consumo de ultraprocessados nessa população. O objetivo foi avaliar se o estresse no trabalho está associado ao consumo de alimentos ultraprocessados por servidores universitários. Trata-se de um estudo realizado com 324 servidores de uma universidade pública. Foram coletados dados sociodemográficos e aplicados dois recordatórios de 24 horas em dias não consecutivos para identificação tanto do consumo de ultraprocessados quanto da contribuição calórica. Ainda, aplicou-se a Escala de Estresse no Trabalho. Utilizou-se regressão de Poisson para verificar associação, com nível de significância de 5\%. O estresse esteve presente em 48,4\% dos servidores, e o consumo foi marcado pela elevada contribuição calórica dos ultraprocessados (30,1\%), além de associação entre maior estresse no trabalho e consumo de ultraprocessados. Essa caracterização abre margem para a criação de políticas públicas que beneficiem a saúde do trabalhador

Palavras-chave: alimentos ultraprocessados, estresse no trabalho, consumo alimentar.

\section{Stress at Work and Association with the Consumption of Ultra-processed Foods by University Workers}

\section{Abstract}

Stress at work increasingly affects workers' health, especially their eating habits. Still, there is a growing level of ultra-processed food consumption in this population. The objective of the study was to assess whether work stress is associated with the consumption of ultra-processed foods by university employees. This is a study carried out with 324 public university employees. Sociodemographic data were collected, and two 24-hour reminders were applied on non-consecutive days to identify both consumption of ultra-processed foods and caloric contribution. Moreover, the Stress at Work scale was applied. Poisson regression was used to verify the association, with a significance level of $5 \%$. Stress was present in $48.4 \%$ of the workers, and consumption was marked by the high caloric contribution of ultra-processed foods $(30.1 \%)$, in addition to an association between greater stress at work and consumption of ultra-processed foods. This characterization paves the way for the creation of public policies that benefit workers' health.

Keywords: ultra-processed foods, occupational stress, food consumption

\section{El Estrés en el Trabajo y Asociación a el Consumo de Alimentos Ultraprocesados por Servidores} Universitários

Resumen

El estrés en el trabajo afecta cada vez más la salud del trabajador, sobre todo sus hábitos alimentarios. Aún, existe un alto consumo de alimentos ultraprocesados en esta población. El objetivo fue evaluar si el estrés laboral está asociado al consumo de alimentos ultraprocesados por parte de los empleados universitarios. Se trata de un estudio realizado con 324 empleados de una universidad pública. Se recolectaron datos sociodemográficos y se aplicaron 2 recordatorios de 24 horas en días no consecutivos para identificar el consumo de alimentos ultraprocesados en cuanto a su aporte calórico. Además, se aplicó la Escala de Estrés en el Trabajo. Se utilizó regresión de Poisson para verificar la asociación, con un nivel de significancia del 5\%. El estrés estuvo presente en el 48,4\% de los servidores, y el consumo estuvo marcado por el alto aporte calórico de los alimentos ultraprocesados $(30,1 \%)$, además de una asociación entre mayor estrés en el trabajo y consumo de alimentos ultraprocesados. Esa caracterización da lugar para la creación de políticas públicas que favorezcan la salud del trabajador.

Palabras clave: alimentos ultraprocesados, estrés ocupacional, consumo de alimentos. 
O mercado de trabalho vem se tornando cada vez mais exigente e competitivo, atribuindo múltiplas demandas (físicas, cognitivas, psíquicas) e resultando em prejuízos na vida psicológica e social dos trabalhadores, em decorrência do estresse ocupacional (Fonseca, 2014). Estima-se que, no Brasil, 72\% dos trabalhadores experimentam, por algum período, condições de estresse relacionadas à atividade laboral (Latorraca , Pacheco, Martimbianco, \& Riera, 2019).

O desgaste ocasionado pelo estresse traz consigo sintomas físicos que prejudicam o desempenho, não só nas atividades relacionadas ao trabalho, mas em suas atividades diárias, gerando assim cansaço, desconforto e reduzindo sua capacidade de manter uma vida equilibrada e saudável (S. Lima, 2017). O contexto laboral pode influenciar o estilo de vida, a prática de atividade física, além de alterações nos hábitos alimentares, trazendo prejuízos para a saúde. Longas jornadas de trabalho, demandas excessivas e exposição a ambientes hostis podem contribuir para a prevalência de excesso de peso na população trabalhadora (Hidalgo et al., 2016; Luckhaupt, Cohen, Li, \& Calvert, 2014).

É neste cenário que emerge o consumo de alimentos ultraprocessados. A falta de tempo para o preparo e consumo de alimentos da sua forma mais natural mobilizou a indústria alimentícia a desenvolver novas técnicas de conservação e de preparo de alimentos, visando a maior praticidade e disponibilizando maior variedade de preparações para consumo rápido (Oliveira et al, 2018).

Os alimentos ultraprocessados são definidos como formulações industriais prontas para o consumo. Este grupo inclui produtos fabricados com vários ingredientes e são produzidos por processos industriais como prensagem, centrifugação, refinação, extração ou mineração, sendo feitos inteiramente ou majoritariamente de substâncias extraídas de alimentos (óleos, gorduras, amido, açúcar, proteínas), derivados de constituintes de alimentos (gorduras hidrogenadas, amido modificado) ou sintetizados em laboratório com base em matérias orgânicas (corantes, aromatizantes, realçadores de sabor) e aditivos usados com função cosmética com o objetivo de modificar as características organolépticas dos produtos (cor, odor, sabor ou textura). Além disso, várias técnicas industriais são usadas na fabricação de produtos ultraprocessados, incluindo extrusão, moldagem e pré-processamento por fritura (Brasil, 2019; Monteiro et al., 2019).

Em sua maioria, apresentam maior conteúdo de açúcar, sódio, gorduras totais e gorduras saturadas e trans, e menor teor de proteínas e fibras, além de promover uma maior ingestão de energia que os alimentos in natura ou minimamente processados. Tais características são fatores de risco para o excesso de peso e associam-se à crescente epidemia de obesidade e doenças crônicas não transmissíveis (DCNT) evidenciadas no Brasil (Louzada et al., 2015; Vale et al., 2019).

$\mathrm{Na}$ literatura pesquisada, cada vez mais se vem estudando a relação entre o consumo excessivo de alimentos ultraprocessados e a sua relação com a presença de doenças crônicas não transmissíveis (Fiolet et al., 2018; Srour et al., 2019; Srour et al., 2020). No entanto são escassos os estudos que evidenciaram associação entre o estresse no trabalho e práticas alimentares (Cattafesta, Zandonade, Bissoli, \& Salaroli, 2019; Reis, Melo, Pinto Júnior, Raposo, \& Munaro, 2016). Especificamente, em relação ao nível de processamento de alimentos, não há estudos que tenham investigado a sua associação com o estresse no trabalho

O objetivo do presente estudo foi avaliar se o estresse no trabalho está associado ao consumo de alimentos ultraprocessados por servidores universitários.

\section{Método}

Realizou-se estudo observacional quantitativo transversal analítico.

\section{Participantes}

A população do estudo foi constituída por todos os servidores (docentes e técnico-administrativos), de ambos os sexos, que possuíam vínculo empregatício numa Universidade Pública Estadual do Ceará, que à época do delineamento da pesquisa somavam 1.522 servidores, sendo 734 (48,2\%) docentes e 788 (51,8\%) funcionários técnico-administrativos.

A amostra foi estimada aplicando-se a fórmula para população finita desenvolvida por Cochran (1977). Considerou-se um erro amostral de 5\% e uma prevalência de doenças cardiovasculares (desfecho considerado no projeto maior) de 29,7\% (Brasil, 2017; Malta et al., 2014) obtendo-se amostra final de 324 servidores. Os servidores foram selecionados de forma não probabilística, estratificada por função, sendo 177 (54,6\%) servidores técnico-administrativos e 147 professores (45,3\%).

\section{Instrumentos}

Foram utilizados três instrumentos: um questionário socioeconômico, demográfico e de saúde, recordatório 24 horas e uma Escala de Estresse no Trabalho, que serão descritos a seguir.

O Questionário socioeconômico, demográfico e laboral continha dados de renda ( $\geq 5$ salários mínimos e $<5$ salários mínimos), escolaridade (sem ensino superior e com ensino superior, pessoas no domicílio ( $\geq 3$ pessoas e $<3$ pessoas), idade ( $<$ de 40 anos e $\geq 40$ anos), sexo (masculino e feminino), estado civil (sem companheiro e com companheiro), categoria de trabalho (docente e servidor técnico administrativo) e vínculo com a instituição (efetivo e terceirizado). O Recordatório Alimentar de $24 \mathrm{~h}$ foi aplicado em dois dias não consecutivos, sendo um dia útil e o outro do final de semana, com intervalo de tempo inferior a um mês em que eram descritos todos os alimentos em medidas caseiras, com auxílio de um álbum de registro fotográfico para facilitar a lembrança das quantidades ingeridas (Zaboto, 1996).

A Escala de Estresse no Trabalho on Job Stress Scale (Karasek \& Theorell, 1990), adaptada e validade para o português por Alves, Chor, Faerrstein e Lopes (2004), possui 17 questões, respondidas em uma escala do tipo Likert de 1 a 4 pontos. As perguntas se referem a características do trabalho, com as seguintes opções de respostas: frequentemente, as vezes, raramente e nunca ou quase nunca; e sobre o ambiente de trabalho, com as seguintes opções de respostas: concordo totalmente / concordo mais que discordo / discordo mais que concordo / discordo totalmente (Alves et al., 2004).

\section{Procedimentos de Coleta de Dados e Cuidados Éticos}

A coleta de dados foi realizada por entrevistadores treinados e após um teste piloto com 30 servidores, escolhidos por conveniência, a fim de testar os instrumentos que foram utilizados na coleta de dados, quanto ao tempo de aplicação e compreensão pela amostra. Esses servidores não fizeram parte da amostra final do presente estudo. “(...) escolhidos por conveniência, a fim de testar os instrumentos que foram utilizados na coleta de.

Os indivíduos que aceitaram participar do estudo assinaram um Termo de Consentimento Livre e Esclarecido, que informava sobre os objetivos, os procedimentos a serem utilizados, os riscos e benefícios inerentes a esta pesquisa. O projeto foi cadastrado na 
Plataforma Brasil com CAAE: 56073116.4.0000.5534 e aprovado pelo Comitê de Ética em Pesquisa da Universidade Estadual do Ceará (UECE) sob parecer de número 1.579.656.

\section{Procedimentos de Análises de Dados}

Os dados alimentares obtidos com o Recordatório Alimentar de $24 \mathrm{~h}$ foram convertidos de medidas caseiras para gramas ou miligramas, por meio de tabela de Pinheiro, Lacerda, Benzecry e Gomes (2008), e analisados quanto ao conteúdo de energia através da Tabela Brasileira de Composição de Alimentos (TACO) do Núcleo de Estudos e Pesquisas em Alimentação (D. M. Lima et al., 2006). Para os alimentos que não estavam disponíveis na TACO, sua composição foi obtida da tabela da POF/IBGE (Brasil, 2011). Os alimentos presentes nos recordatórios foram listados e estratificados e as preparações foram desmembradas em ingredientes em uma planilha do Excel em quatro grupos: alimentos in natura ou minimamente processados; ingredientes culinários processados; alimentos processados; e alimentos ultraprocessados, seguindo a classificação NOVA (Monteiro et al., 2019). Quando não foi possível desmembrar a preparação, considerou-se o ingrediente em maior quantidade e sua classificação.

Calculou-se o percentual da participação calórica (\%PC) de cada grupo em relação ao valor calórico médio total (VCMT) consumido pelo indivíduo, a partir da seguinte equação: “\%PC, 2011 $=($ caloria provenientes de cada grupo*100) / calorias totais da dieta". Os indivíduos foram categorizados em quintis de distribuição para a contribuição calórica dos alimentos ultraprocessados (\%PC). Para fins de análise, considerou-se maior consumo o quinto quintil e menor consumo os quintis 1 a 4 .

As questões da Escala de Estresse no Trabalho são divididas em três dimensões: demanda psicológica, controle e suporte. Após a obtenção dos escores medianos de cada domínio, cada dimensão foi categorizada em: demandas, controle e suporte social para aqueles com pontuação menor ou igual à mediana, ou demandas altas, controle e suporte social para aqueles com pontuação parcial maior que a mediana. (Alves et al., 2004).

Para efeito de interpretação, uma categoria foi combinada com base no modelo Demanda-Controle (Alves et al., 2004), a dimensão estresse geral ,considerou os trabalhos de alta exigência (alta demanda e baixo controle), como sendo o grupo com maior exposição à tensão no trabalho.

Os escores de cada domínio foram somados, e a mediana foi utilizada como ponto de corte para categorização, uma vez que não existem valores estabelecidos na literatura. A classificação foi assim estabelecida: domínio psicológico (baixo $<14$ ou alto $>14$ ); tomada de decisões (alto $>18$ ou baixo $<18$ ); apoio social (alto $>$ 9 ou baixo $<9$ ); e estresse geral (baixo $\leq 41$ ou alto $>41$ ).

Realizou-se uma análise descritiva, sendo as variáveis numéricas descritas em medianas e intervalo interquartil, e as categóricas, em frequências simples e percentuais. As características dos indivíduos foram apresentadas comparando-se aqueles com menor consumo (quintis 1 a 4) vs. maior consumo (quintil 5) de alimentos ultraprocessados, utilizando-se o teste do Qui-quadrado. O teste de normalidade usado foi de Kolmogorov-Smirnov.

Para investigar associação entre o estresse e o consumo de ultraprocessados, utilizou-se análise de regressão de Poisson, com estimativa robusta da variância, sem e com ajuste para variáveis de confusão (variáveis socioeconômicas e demográficas). Entraram no modelo ajustado apenas as variáveis que apresentaram $p<0,20$ no modelo não ajustado. Considerou-se como variáveis independentes os domínios/escalas de estresse, e a variável desfecho foi o consumo de alimentos ultraprocessados (menor e maior consumo). Os dados foram analisados no programa estatístico STATA, versão 13.0, adotando-se o nível de significância de 5\% para todos os testes.

\section{Resultados}

O grupo de servidores estudado era predominantemente do sexo feminino $(67,0 \%)$, tinha 40 anos ou mais $(56,8 \%)$, pardos, pretos ou amarelos $(60,8 \%)$, vivia com companheiro (50,6\%), tinha renda familiar acima de cinco salários mínimos (53,1\%), e possuía nível superior $(73,1 \%$ ) (Tabela 1$)$.

O consumo calórico total mediano foi de 1.721,6 (1.390,3 $2.018,8) \mathrm{Kcal}$, sendo $30,1 \%(19,7$ - 40,2) proveniente do consumo de alimentos ultraprocessados. O consumo de ultraprocessados foi maior entre os servidores com idade $<40$ anos $(p=0,001)$, vivendo sem companheiro $(p=0,039)$, que exerciam funções técnico-administrativas $(p=0,011)$ e eram efetivos $(p=0,001)(\mathrm{Ta}-$ bela 1).

Tabela 1

Características sociodemográficas e laborais, segundo o consumo de alimentos classificados pelo nivel de processamento. Fortaleza, 2017.

\begin{tabular}{|c|c|c|c|c|}
\hline \multirow[b]{2}{*}{$\begin{array}{c}\text { Variáveis } \\
\text { sociodemográficas }\end{array}$} & \multicolumn{2}{|c|}{ Total } & \multicolumn{2}{|c|}{ Ultra processados } \\
\hline & $N$ & $\%$ & $\begin{array}{c}\text { Menor } \\
\text { consumo } \\
n(\%)\end{array}$ & $\begin{array}{c}\text { Maior } \\
\text { consumo } \\
n(\%)\end{array}$ \\
\hline Sexo & & $=0,968$ & & \\
\hline Masculino & 107 & 33,0 & $86(80,4)$ & $21(19,6)$ \\
\hline Feminino & 217 & 67,0 & $174(80,2)$ & $43(19,8)$ \\
\hline Idade & & $=0,001$ & & \\
\hline$<40$ anos & 140 & 43,2 & $101(72,1)$ & $39(27,9)$ \\
\hline$\geq 40$ anos & 184 & 56,8 & $159(86,4)$ & $25(13,6)$ \\
\hline Raça & & $=0,405$ & & \\
\hline $\begin{array}{l}\text { Pardos, Pretos e } \\
\text { Amarelos }\end{array}$ & 197 & 60,8 & $161(81,7)$ & $36(18,3)$ \\
\hline Branca & 127 & 39,2 & $99(78,0)$ & $28(22,0)$ \\
\hline Estado civil & & $=0,039$ & & \\
\hline Sem companheiro & 160 & 49,4 & $121(75,6)$ & $39(24,3)$ \\
\hline Com companheiro & 164 & 50,6 & $139(84,8)$ & $25(15,2)$ \\
\hline Renda familiar & & $=0,164$ & & \\
\hline$\geq 5 \mathrm{SM}^{*}$ & 172 & 53,1 & $143(83,1)$ & $29(16,9)$ \\
\hline$<5 \mathrm{SM}$ & 152 & 46,9 & $117(77,0)$ & $35(23,0)$ \\
\hline Pessoas dom*** & & $=0,282$ & & \\
\hline$\geq 3$ pessoas & 146 & 45,1 & $121(82,9)$ & $25(17,1)$ \\
\hline$<3$ pessoas & 178 & 54,9 & $139(78,1)$ & $39(21,9)$ \\
\hline Escolaridade & & $=0,067$ & & \\
\hline Sem ensino superior & 87 & 26,9 & $64(73,6)$ & $23(26,4)$ \\
\hline Com ensino superior & 237 & 73,1 & $196(82,7)$ & $41(17,3)$ \\
\hline Categoria & & & $\mathrm{p}=0,011$ & \\
\hline Docente & 147 & 45,4 & $127(86,4)$ & $20(13,6)$ \\
\hline $\begin{array}{l}\text { Servidor técnico } \\
\text { administrativo. }\end{array}$ & 177 & 54,6 & $133(75,1)$ & $44(24,9)$ \\
\hline Vinculo UECE & & $=0,001$ & & \\
\hline Efetivo & 147 & 45,4 & $130(88,4)$ & $17(11,6)$ \\
\hline Terceirizado & 177 & 54,6 & $130(73,5)$ & $47(26,5)$ \\
\hline
\end{tabular}

Nota. *: SM: salário mínimo. Menor consumo: $1^{\circ}$ ao $4^{\circ}$ quintil. Maior consumo: $5^{\circ}$ quintil. **: Teste do Qui-quadrado. ***: domicílio.

Analisando-se o estresse no trabalho, verificou-se alta demanda psicológica em 40,4\%, baixo controle para habilidades/ decisões em $51,2 \%$ e baixo apoio social em 56,8\% dos analisados. O alto estresse no trabalho geral, baseado no Modelo Demanda-Controle, esteve presente em 48,4\% dos servidores. 
Tabela 2

Contribuição calórica de ultraprocessados por dimensões de estresse no trabalho, Fortaleza, 2017.

\begin{tabular}{|c|c|c|}
\hline Dimensão & \multicolumn{2}{|c|}{$\begin{array}{l}\text { Contribuição calórica de } \\
\text { ultraprocessados }\end{array}$} \\
\hline Demanda (psicológica) para o trabalho ${ }^{1}$ & $\%$ & Valor de $p$ \\
\hline Baixa & $30,0(19,8-40,8)$ & \multirow{2}{*}{$p=0,964$} \\
\hline Alta & $30,1(19,7-40-1)$ & \\
\hline \multicolumn{3}{|l|}{ Controle para habilidade/decisões ${ }^{2}$} \\
\hline Alto & $31,9(21,7-44,2)$ & \multirow{2}{*}{$p=0,037$} \\
\hline Baixo & $27,9(19,1-36,4)$ & \\
\hline \multicolumn{3}{|l|}{ Apoio social (relações sociais) ${ }^{3}$} \\
\hline Alto & $29,3(18,3-39,9)$ & \multirow{2}{*}{$p=0,521$} \\
\hline Baixo & $31,3(20,2-40,8)$ & \\
\hline \multicolumn{3}{|l|}{ Estresse no trabalho (geral) ${ }^{4}$} \\
\hline Baixo & $30,0(19,8-40,0)$ & \multirow{2}{*}{$p=0,879$} \\
\hline Alto & $30,1(18,9-40,2)$ & \\
\hline
\end{tabular}

Nota. Teste Mann Whitney. 1: Indivíduos na categoria de alto consumo em cada categoria de escala do estresse. 2: Alta demanda: alto desgaste psicológico. Baixa demanda: baixo desgaste psicológico. 3: Baixo controle: indivíduo passivo. Alto controle: indivíduo ativo. 4: Baixo apoio social: maior desgaste. Alto apoio social: menor desgaste.

A Tabela 3 apresenta a associação do estresse no trabalho com alto consumo de ultraprocessados, sendo verificado que indivíduos com maior estresse geral no trabalho apresentaram maior consumo de alimentos ultraprocessados, quando comparados aos indivíduos de menor estresse $[\mathrm{RP}=1,19$ (IC95\% = 1,02 - 1,39)]. Para as três escalas específicas de estresse (Demanda para o trabalho, Controle para habilidade/decisões, e Apoio social), não houve associação.

Tabela 3

Modelo não ajustado e ajustado da regressão de Poisson para a associação do estresse com o maior consumo de produtos ultraprocessados. Fortaleza, 2017.

\begin{tabular}{|c|c|c|c|}
\hline \multirow[b]{2}{*}{ Escala do Estresse } & \multirow[b]{2}{*}{$n(\%)^{1}$} & \multicolumn{2}{|c|}{ Maior consumo de ultraprocessados } \\
\hline & & $\begin{array}{c}\text { NA RP (IC } \\
95 \%)\end{array}$ & $\begin{array}{c}\text { Ajustado RP (IC } \\
95 \%)\end{array}$ \\
\hline $\begin{array}{l}\text { Demanda (psicológica) } \\
\text { para o trabalho }\end{array}$ & & 0,988 & 0,887 \\
\hline Baixa & $193(59,6)$ & Referência & Referência \\
\hline Alta & $131(40,4)$ & $1,00(0,90-1,11)$ & $0,99(0,88-1,11)$ \\
\hline $\begin{array}{l}\text { Controle para habilidade/ } \\
\text { decisões }\end{array}$ & & 0,048 & 0,508 \\
\hline Alto & $158(48,8)$ & Referência & Referência \\
\hline Baixo & $166(51,2)$ & $1,10(1,00-1,23)$ & $1,04(0,92-1,18)$ \\
\hline $\begin{array}{l}\text { Apoio social (relações } \\
\text { sociais) }\end{array}$ & & 0,531 & 0,945 \\
\hline Alto & $154(43,2)$ & Referência & Referência \\
\hline Baixo & $170(56,8)$ & $0,97(0,87-1,07)$ & $1,00(0,88-1,13)$ \\
\hline $\begin{array}{l}\text { Estresse no trabalho } \\
\text { (geral) }\end{array}$ & & 0,639 & 0,026 \\
\hline Baixo & $167(51,6)$ & Referência & Referência \\
\hline Alto & $157(48,4)$ & $1,02(0,92-1,14)$ & $1,19(1,02-1,39)$ \\
\hline
\end{tabular}

Nota. $n$ : número absoluto; NA: Não ajustado; RP: Razão de prevalências; IC: Intervalo de confiança. *: Entraram no modelo ajustado apenas as variáveis que apresentaram $p<0,20$ no modelo não ajustado

\section{Discussão}

Foi alto o consumo de alimentos ultraprocessados nos servidores estudados e esteve associado à presença de estresse geral no trabalho, além disso a contribuição calórica dos alimentos ultraprocessados na dieta dos servidores mostrou-se superior à média da população brasileira, segundo dados da Pesquisa de Orçamentos Familiares de 2017-2018, apontam que 19,7\% das calorias ingeridas são provenientes de alimentos ultraprocessados. Além disso 32,8\% das despesas com a alimentação são destinadas para alimentação fora de casa (Brasil, 2019; Brasil, 2020).

Para Oliveira et al. (2018), a incorporação destes alimentos é facilmente verificável, independentemente da idade, classe social e atividade profissional. Além de ser uma prática cada vez mais adotada no ambiente doméstico, bem como nos espaços que agregam pessoas, sobretudo no ambiente de trabalho.

Nas últimas décadas, o ato de se alimentar se modificou, pelo pouco tempo destinado ao preparo, escolha e consumo dos alimentos. Isso pode ser observado no cotidiano de muitos trabalhadores que possuem sobrecarga de tarefas, metas a serem alcançadas e jornadas de trabalho extenuantes (Araújo, Costa-Souza, \& Trad, 2010).

Os trabalhadores passam a maior parte do tempo no local de trabalho, tendo suas escolhas alimentares influenciadas pelo tempo e disponibilidade alimentar, além do pouco tempo para a realização dessas refeições. Muitas vezes, renunciam a uma alimentação mais saudável e optam por lanches rápidos e refeições práticas (Cattafesta et al., 2019)

Grande parte das pesquisas que envolvem o consumo alimentar do trabalhador verificam que alimentos tradicionais da dieta do brasileiro como o arroz e feijão, bem como frutas e hortaliças aparecem cada vez menos na rotina alimentar desse trabalhador, dando lugar aos alimentos ultraprocessados e lanches do tipo fast food (Fröhlich et al., 2019). Isto pode ser explicado pelo fato da indústria alimentícia disponibilizar cada vez mais opções de alimentos de fácil preparo e alto valor calórico (Tonini, Broll, \& Corrêa, 2013), somado à alta carga horária de trabalho, que leva os servidores a buscarem tais produtos (Cattafesta et al., 2019).

Os alimentos ultraprocessados apresentam elevada densidade energética por maior teor de açúcar livre e gorduras saturadas em comparação aos alimentos in natura e minimamente processados, além de baixo teor de fibras, proteínas, vitaminas e minerais (Monteiro et al, 2019), e pode levar a importantes implicações sobre a saúde dos trabalhadores como Diabetes tipo 2, (Srour et al., 2019), obesidade (Beslay et al., 2020), risco cardiovascular (Srour et al., 2020), além do risco de alguns tipos de neoplasias (Fiolet et al., 2018). Ainda, para Ulrich-Lai, Fulton, Wilson, Petrovich e Rinaman (2015), a exposição ao estresse pode causar alterações qualitativas e quantitativas no padrão alimentar, sobretudo nos trabalhadores que são expostos a intensa jornada de trabalho, explicando grande parte dessas alterações (Cattafesta et al., 2019).

No presente estudo, indivíduos com maior estresse geral no trabalho apresentaram maior consumo de alimentos ultraprocessados. Também Penaforte, Matta e Japur (2016), que avaliaram a associação entre estresse, comportamento alimentar e consumo alimentar em estudantes universitários, encontraram que o consumo de salgados e lanches prontos para consumo/fast food, bem como alimentos ricos em açúcar foi frequente em indivíduos com maior estresse.

A exposição ao estresse pode provocar uma tendência de consumo desses alimentos, com alta densidade energética, além do excesso de açúcar, sódio e de gorduras trans, tendo muitas vezes como consequência excesso de peso e DCNT (Pecorato, Reyes, Gomez, Bhargava, \& Dallman, 2004; Zellner et al., 2006).

Evidências sugerem que o estresse altera o comportamento alimentar, redirecionando as escolhas alimentares para alimentos com maior palatabilidade, especialmente os ricos em açúcar e gordura, como os ultraprocessados (Penaforte et al., 2016).

Para Dalmazo et al. (2019), o estresse crônico se associa com a maior preferência por esse tipo de alimentos, pela maior exposição ao cortisol, a dopamina, a leptina e a insulina, que atuam no nível central, estimulando o desejo por alimentos palatáveis e 
que geram prazer e emoções positivas. Desta forma, as escolhas alimentares são utilizadas na tentativa de utilizar o alimento como forma de conforto e "automedicação".

Essa pesquisa apresenta algumas limitações que precisam ser consideradas. A primeira é o próprio delineamento transversal, que impossibilita avaliar a relação causa-efeito, além do uso da Escala de Estresse no Trabalho ou Job Stress Scale, que não leva em consideração as transformações significativas ocorridas recentemente no mundo do trabalho e das organizações. Outra limitação é inerente a todos os métodos de avaliação do consumo alimentar como o viés de memória, sub ou superrelatos e erros nas estimativas de porções dos alimentos mencionados, no entanto, foram tomadas medidas para minimizar, como o treinamento dos pesquisadores e uso de instrumentos padronizados.

Contudo, a pesquisa apresenta uma amostra representativa de servidores e docentes, de ambos os sexos, de uma universidade pública como ponto forte. Aborda ainda uma temática relevante, de destaque, em que se investigou o consumo de alimentos ultraprocessados e sua associação com o estresse no trabalho, cujas evidências ainda são escassas. Por fim, os servidores apresentaram considerável contribuição calórica provenientes dos alimentos ultraprocessados, que esteve diretamente associada ao maior estresse no trabalho.

A caracterização do perfil de saúde dos servidores a partir de informações do ambiente de trabalho, buscando investigar estresse e fatores associados aos aspectos alimentares, é importante para que sejam elaboradas estratégias que melhorem as condições de trabalho. Além disso, investigar o consumo alimentar por meio do grau de processamento dos alimentos, cada vez mais, se torna uma temática abordada na literatura cientifica, para a criação de políticas que beneficiem a saúde do trabalhador.

\section{Referências}

Alves, M. G. M., Chor, D., Faerrstein, E. \& Lopes, C. S. (2004). Versão resumida da "job stress scale": adaptação para o português. Rev. Saúde Pública, 38(2), 164-171. https://doi.org/10.1590/S0034-89102004000200003

Araújo, M.P.N., Costa-Souza, J., \& Trad, L.A.B. (2010). A alimentação do trabalhador no Brasil: um resgate da produção científica nacional. Hist. cienc. saude-Manguinhos, 17(4), 975-992. https://doi.org/10.1590/S010459702010000400008

Beslay, M., Srour, B., Méjean, C., Allès, B., Fiolet, T., Debras, C., . . \& \&Touvier, M. (2020). Intake of ultra-processed foods in association with changes in BMI and risk of overweight and obesity: a prospective analysis of the French NutriNet-Santé cohort. PLoS Med, 17(8). https://doi.org/10.1371/journal. pmed.1003256

Brasil (2017). Vigitel Brasil 2016: Vigilancia de fatores de risco e protecão para doenças crônicas por inquérito telefônnico: estimativas sobre frequência e distribuição sociodemográfica de fatores de risco e proteção para doenças crônicas nas capitais dos 26 estados brasileiros e no Distrito Federal em 2016. Brasília: Ministério da Saúde, 160p. Recuperado de https://portalarquivos2.saude.gov.br/images/ pdf/2018/marco/02/vigitel-brasil-2016.pdf

Brasil (2019). Pesquisa de orçamentos familiares 2017-2018 : primeiros resultados / IBGE, Coordenacão de Trabalbo e Rendimento. - Rio de Janeiro: IBGE, 69. Recuperado de https://biblioteca.ibge.gov.br/visualizacao/livros/liv101670.pdf

Brasil (2020). Pesquisa de orcamentos familiares 2017-2018 : análise do consumo alimentar pessoal no Brasil / IBGE, Coordenação de Trabalho e Rendimento. - Rio de Janeiro: IBGE, 120. Recuperado de https://biblioteca.ibge.gov.br/visualizacao/ livros/liv101742.pdf

Cattafesta, M., Zandonade, E., Bissoli, N. S., \& Salaroli, L. B. (2019). Padrões alimentares de trabalhadores bancários e sua associação com fatores socioeconômicos, comportamentais e laborais. Ciênc. saúde coletiva, Rio de Janeiro, 24(10), 3909-3922. https://doi.org/10.1590/1413$\underline{812320182410.31342017}$

Dalmazo, A. L., Fetter, C., Goldmeier, S., Irigoyen, M. C., Pellanda, L. C., Barbosa, E. C. D., . . \& Osório, D. R. D. (2019). Estresse e Consumo Alimentar em Pacientes Hipertensos. Arq. Bras. Cardiol., 113(3), 374-380. https://doi.org/10.5935/abc. 20190175

Fiolet, T., Srour, B., Sellem, L., Kesse-Guyot, E., Allès, B., Mèjean, C., . . \& \& Touvier, M. (2018). Consumption of ultra-processed foods and cancer risk: results from NutriNet-Santé prospective cohort. BMJ, 360, k322. https:// doi.org/10.1136/bmj.k322
Fonseca, I. S. S., \& Araújo, T. M. (2014). Prevalência de transtornos mentais comuns em industriários da Bahia. Rev Bras Saúde Ocup, 39(129), 35-49. https://doi.org/10.1590/0303-7657000065012

Fröhlich, C., Garcez, A., Canuto, R., Paniz, V. M. V., Pattussi, M. P., \& Olinto, M. T. A. (2019). Obesidade abdominal e padrões alimentares em mulheres trabalhadoras de turnos. Ciênc. saúde coletiva, 24(9), 3283-3292. https://doi. org/10.1590/1413-81232018249.27882017

Hidalgo, K. D., Mielke, G. I., Parra, D. C., Lobelo, F., Simões, E. D., Gomes, G. O., . \& \& Hallal, P. C. (2016). Health promoting practices and personal lifestyle behaviors of Brazilian health professionals. BMC Public Health, 16(1),1114. https://doi.org/10.1186/s12889-016-3778-2

Karasek, R., \& Theorell, T. (1990). Healthy work: stress, productivity and the reconstruction of working life. New York: Basic Books. https://doi. org/10.1177/002218569103300112

Latorraca, C. O. C., Pacheco, R. L., Martimbianco, A. L. C., \& Riera, R. (2019). O que as revisões sistemáticas Cochrane dizem sobre prevenção e tratamento da síndrome de burnout e estresse no trabalho? Diagn. Tratamento, 24(3), 119-125. Recuperado de http://docs.bvsalud.org/ biblioref/2019/12/1026704/rdt v24n3 119-125.pdf

Lima, D. M., Colugnati, F. A. B., Padovani, R. M., Rodriguez-Amava, D. B., Salay, E., \& Galeazzi, M. A. M. (2006). Tabela brasileira de composição de alimentos: versão II. Campinas: NEPA/UNICAMP.

Lima, S. (2017). Indisciplina em sala de aula: concepções, interação social e ação docente-uma análise qualitativa. CLAIQ , 1, 836-846. Recuperado de https:// proceedings.ciaiq.org/index.php/ciaiq2017/article/view/1404

Louzada, M. L. C., Martins, A. P. B., Canella, D. S., Baraldi, L. G., Levy, R. B., Claro, R. M., ... \& Monteiro, C. A. (2015). Alimentos ultraprocessados e perfil nutricional da dieta no Brasil (2008-2009). Rev de Saú Públ, 49(38), 1-10. https://doi.org/10.1590/S0034-8910.2015049006132

Luckhaupt, S. E., Cohen, M. A., Li, J., \& Calvert, G. M. (2014). Prevalence of obesity among U.S. workers and associations with occupational factors. $\mathrm{Am}$ J Prev Med, 46(3), 237-248. https://doi.org/ 10.1016/j.amepre.2013.11.002

Malta, D. C., Bernal, R. T. I., Nunes, M. L., Oliveira, M. M., Iser, B. P. M., Andrade, S. S. C. A., . . \& Silva Júnior, J. B. (2014). Prevalência de fatores de risco e proteção para doenças crônicas não transmissíveis em adultos: estudo transversal, Brasil 2012. Epidemiologia e Serviços de Saúde, 23(4), 609622. https://doi.org/10.5123/S1679-49742014000400003

Martins, A. P., Levy, A. P., Claro, R. M., Moubarac, J. C., \& Monteiro, C. A. (2013). Increased Contribution of ultra-processed food products in the Brazilian diet (1987-2009). Rev de Saú Públ, 47(4), 656-665. https://doi. org/10.1590/S0034-8910.2013047004968

Monteiro, C. A., Cannon, G., Levy, R. B., Moubarac, J., Louzada, M. L., Rauber, F., .. \& \& Jaime, P. C. (2019). Ultra-processed foods: what they are and how to identify them. Public Health Nutr, 22(5), 936-941. https://doi. org/10.1017/S1368980018003762

Moura, D. C. A., Greco, R. M., Paschoalin, H. C., Portela, L. F., Arreguy-Sena, C., \& Chaoubah, A. (2018). Demandas psicológicas e controle do processo de trabalho de servidores de uma universidade pública. Ciênc. saúde coletiva, 23(2), 481-490. https://doi.org/10.1590/1413-81232018232.13892015

Oliveira, E. I. A. D., Melo, L. M., Graciliano, N. G., Almeida, A. A. S., Maia, D. E. R. S., Araujo, J. O., . . \& Vasconcelos, S. M. L. (2018). Consumo de alimentos ultraprocessados nos lanches de servidores do HUPAA da UFAL, Maceió, Alagoas. GEPNEWS, 2(1), 25-34. Recuperado de http://www.seer. ufal.br/index.php/gepnews/article/view/7720/5542

Pecorato, N., Reyes, F., Gomez, F., Bhargava, A., \& Dallman, M. F. (2004). Chronic stress promotes palatable feeding, which reduces signs of stress: feedforward and feedback effects of chronic stress. Endocrinology, 145(8) 3754-3762. https://doi.org/10.1210/en.2004-0305

Penaforte, F. R. O., Matta, N. C., \& Japur, C. C. (2016). Associação entre estresse e comportamento alimentar em estudantes universitários. Demetra, 11(1), 225-237. https://doi.org/10.12957/demetra.2016.18592

Pinheiro, A. B. V., Lacerda, E. M. A., Benzecry, E. H., \& Gomes, M. C. S. (2008) Tabela para avaliação de consumo alimentar em medidas caseiras $\left(5^{\mathrm{a}} \mathrm{ed}\right)$. São Paulo: Atheneu, 131p

Reis, M. C., Melo, N. S. A., Pinto Junior, E. P., Raposo, M. T., \& Munaro, H. L. R. (2016). Condições de saúde e fatores associados a satisfação com a vida em acadêmicos de Fisioterapia. Revista Saúde.com, 12(3), 638-645. Recuperado de http://www.repositorio.ufc.br/bitstream/riufc/22373/1/2016 art mcreis. pdf

Srour, B., Fezeu, L. K., Kesse-Guyot, E., Allés, B., Debras, C., Druesne-Pecollo, N., . . \& Touvier, M. (2020). Ultraprocessed Food Consumption and Risk of Type 2 Diabetes Among Participants of the NutriNet-Sante Prospective Cohort. JAMA Intern Med, 180(2), 283-291. https://doi.org/10.1001/ jamainternmed.2019.5942

Srour, B., Fezeu, L. K., Kesse-Guyot, E., Allés, B., Mèjean, C., Andrianasolo, R. M., .. \& \& Touvier, M. (2019). Ultra-processed food intake and risk of cardiovascular disease: prospective cohort study (NutriNet-Santé). BMJ, 365, 11451. https://doi.org/10.1136/bmj.11451 
Tonini, E., Broll, A. M., \& Corrêa, E. N. (2013). Avaliação do estado nutricional e hábito alimentar de funcionários de uma instituição de ensino superior do oeste de Santa Catarina. O Mundo da Saúde, 37(3), 268-279. Recuperado de http://bvsms.saude.gov.br/bvs/artigos/mundo saude/avaliacao estado nutricional habito alimentar.pdf

Ulrich-Lai, Y. M., Fulton, S., Wilson, M., Petrovich, G., \& Rinaman, L. (2015). Stress exposure, food intake and emotional state. Stress, 18(4), 381-399.

Vale, D., Morais, C. M. M., Pedrosa, L. F. C., Ferreira, M. A. F., Oliveira, A G. R. C., \& Lyra, C. O. (2019). Correlação espacial entre o excesso de peso, aquisição de alimentos ultraprocessados e o desenvolvimento humano no Brasil. Ciência \& Saúde Coletiva, 24(3), 983-996. https://doi. org/10.1590/1413-81232018243.35182016

Zaboto, C. B. (1996). Registro fotográfico para inquéritos dietéticos: utensílios e porções. Goiânia: UFG.

Zellner, D. A., Loaiza, S., Gonzalez, Z., Morales, J., Pecora, D. \& Wolf, A. (2006). Food selection changes under stress. Physiol Behav., 87(4), 189-193. https:// doi.org/10.1016/j.physbeh.2006.01.014

Informações sobre as autoras:

\section{Bianca de Oliveira Farias}

Universidade Estadual do Ceará

Av. Dr. Silas Munguba, 1700 - Itaperi, 60714-903 Fortaleza, CE, Brasil

E-mail: biancafariasnutri@gmail.com

Ana Carolina Montenegro Cavalcante

E-mail: carolyna4481@hotmail.com

Rafaella Maria Monteiro Sampaio

E-mail: rafaellammsampaio@gmail.com

Maria Yasmin Paz Teixeira

E-mail: yasmin_acop@hotmail.com

Helena Alves de Carvalho Sampaio

E-mail: dr.hard2@gmail.com

\section{Soraia Pinheiro Machado}

E-mail:soraia.arruda@uece.br 